\title{
Genome sequence of Rhizobium leguminosarum bv trifolii strain WSM1689, the microsymbiont of the one flowered clover Trifolium uniflorum
}

\author{
Jason Terpolilli', Tian Rui ${ }^{1}$, Ron Yates ${ }^{1,2}$, John Howieson ${ }^{1}$, Philip Poole ${ }^{3,4}$, Christine Munk ${ }^{5}$, \\ Roxanne Tapia ${ }^{5}$, Cliff Han ${ }^{5}$, Victor Markowitz ${ }^{6}$, Reddy Tatiparthi ${ }^{7}$, Konstantinos \\ Mavrommatis $^{6}$, Natalia Ivanova ${ }^{7}$, Amrita Pati ${ }^{7}$, Lynne Goodwin ${ }^{5}$, Tanja Woyke ${ }^{7}$, Nikos \\ Kyrpides $^{7}$ \& Wayne Reeve ${ }^{* 1}$. \\ ${ }^{1}$ Centre for Rhizobium Studies, Murdoch University, Perth, Australia \\ 2 Department of Agriculture and Food, Western Australia, Australia \\ ${ }^{3}$ Department of Plant Sciences, University of Oxford, UK \\ ${ }^{4}$ Sir Walter Murdoch Adjunct Professor, Murdoch University, Perth, Australia \\ ${ }^{5}$ Los Alamos National Laboratory, Bioscience Division, Los Alamos, New Mexico, USA \\ ${ }^{6}$ Biological Data Management and Technology Center, Lawrence Berkeley National \\ Laboratory, Berkeley, California, USA \\ ${ }^{7}$ DOE Joint Genome Institute, Walnut Creek, California, USA
}

*Correspondence: Wayne Reeve (W.Reeve@murdoch.edu.au)

Keywords: root-nodule bacteria, nitrog en fixation, lupin-nodulating, rhizobia, Alphaproteobacteria

Rhizobium leguminosarum bv. trifolii is a soil-inhabiting bacterium that has the capacity to be an effective $\mathrm{N}_{2}$-fixing microsymbiont of Trifolium (clover) species. $R$. leguminosarum bv. trifolii strain WSM1689 is an aerobic, motile, Gram-negative, non-spore-forming rod that was isolated from a root nodule of Trifolium uniflorum collected on the edge of a valley $6 \mathrm{~km}$ from Eggares on the Greek Island of Naxos. Although WSM1689 is capable of highly effective $\mathrm{N}_{2}$-fixation with $T$. uniflorum, it is either unable to nodulate or unable to fix $\mathrm{N}_{2}$ with a wide range of both perennial and annual clovers originating from Europe, North America and Africa. WSM1689 therefore possesses a very narrow host rang e for effective $\mathrm{N}_{2}$ fixation and can thus play a valuable role in determining the geographic and phenological barriers to symbiotic performance in the genus Trifolium. Here we describe the features of $R$. leguminosarum bv. trifolii strain WSM1689, tog ether with the complete genome sequence and its annotation. The 6,903, 379 bp genome contains 6,709 protein-coding genes and 89 RNA-only encoding genes. This multipartite genome contains six distinct replicons; a chromosome of size 4,854,518 bp and five plasmids of size $667,306,518,052,341,391,262,704$ and 259,408 bp. This rhizobial genome is one of 20 sequenced as part of a DOE Joint Genome Institute 2010 Community Sequencing Prog ram.

\section{Introduction}

The nitrogen $(\mathrm{N})$ cycle is one of the most important biogeochemical processes underpinning the existence of life on Earth. A key step in this cycle is to convert relatively inert atmospheric dinitrogen $\left(\mathrm{N}_{2}\right)$ into a bioaccessible form such as ammonia $\left(\mathrm{NH}_{3}\right)$ through a process referred to as biological nitrogen fixation (BNF). BNF is performed only by a specialized subset of Bacteria and Archaea that possess the necessary cellular machinery to enzymatically reduce $\mathrm{N}_{2}$ into $\mathrm{NH}_{3}$.
Some of these bacteria (termed rhizobia or root nodule bacteria) have evolved non-obligatory symbiotic relationships with legumes whereby the bacteria receive a carbon source from the plant and in return supply fixed $\mathrm{N}$ to the host [1]. Harnessing this association can boost soil $\mathrm{N}$-inputs and therefore production yields of legumes, or non-legumes grown in subsequent years, without the need for supplementation with industrially synthesized N-based fertilizers [2]. 
Some of the most widely cultivated pasture legumes are members of the legume genus Trifolium (clover). The natural distribution of these species spans three centers of diversity, with an estimated $28 \%$ of species in the Americas, $57 \%$ in Eurasia and 15\% in sub-Saharan Africa [3]. Approximately 30 species of clover, predominately of Eurasian origin, are widely grown as annual and perennial species in pasture systems in Mediterranean and temperate climatic zones [3]. Globally-important perennial species of clover include T. repens (white clover), T. pratense (red clover), $T$. fragiferum (strawberry clover) and $T$. hybridum (alsike clover). While clovers are known to form $\mathrm{N}_{2}$-fixing symbiotic associations with Rhizobium leguminosarum bv. trifolii, there exists wide variation in symbiotic compatibility across different strains and hosts from ineffective (non- $\mathrm{N}_{2}$-fixing) nodulation to fully effective $\mathrm{N}_{2}$-fixing partnerships.

Rhizobium leguminosarum bv. trifolii strain WSM1689 was isolated in 1995 from a nodule of the peren nial clover Trifolium uniflorum collected on the edge of a valley $6 \mathrm{~km}$ from Eggares on the Greek Island of Naxos. T. uniflorum is one of small number of perennial Trifolium spp. found in the dry, Mediterranean basin. While WSM1689 has been shown to be either ineffective or unable to nodulate a range of annual and perennial Trifolium sp., it is a highly effective $\mathrm{N}_{2}$-fixing microsymbiont of T. uniflorum [4]. Therefore, $R$. leguminosarum bv. trifolii WSM1689 has a very narrow host range and thus represents a good isolate to study the genetic basis of symbiotic specificity. The availability of this sequence data also complements the already published genomes of the clover-nodulating $R$. leguminosarum bv. trifolii WSM1325 [5] and WSM2304 [6]. Here we present a summary classification and a set of general features for $R$. leguminosarum bv. trifolii strain WSM1689 together with the description of the complete genome sequence and its annotation.

\section{Classification and features}

R. leguminosarum bv. trifolii strain WSM1689 is a motile, non-sporulating, non-encapsulated, Gramnegative rod in the order Rhizobiales of the class Alphaproteobacteria. The rod-shaped form varies in size with dimensions of approximately 0.25-0.5 $\mu \mathrm{m}$ in width and $2.0 \mu \mathrm{m}$ in length (Figure $1 \mathrm{Left}$ and 1 Center). It is fast growing, forming colonies within 3-4 days when grown on half strength Lupin Agar ( $1 / 2 \mathrm{LA}$ ) [7], tryptone-yeast extract agar (TY) [8] or a modified yeast-mannitol agar (YMA) [9] at $28^{\circ} \mathrm{C}$. Colonies on $1 / 2 \mathrm{LA}$ are opaque, slightly domed and moderately mucoid with smooth margins (Figure 1 Right). Minimum Information about the Genome Sequence (MIGS) is provided in Table 1.

Figure 2 shows the phylogenetic neighborhood of R. leguminosarum bv. trifolii strain WSM1689 in a 16S rRNA gene sequence based tree. This strain shares 100\% (1362/1362 bp) sequence identity to the 16S rRNA gene of $R$. leguminosarum bv. trifolii strain WSM1325 [5] and R. leguminosarum bv. trifolii strain WSM2304 [6].
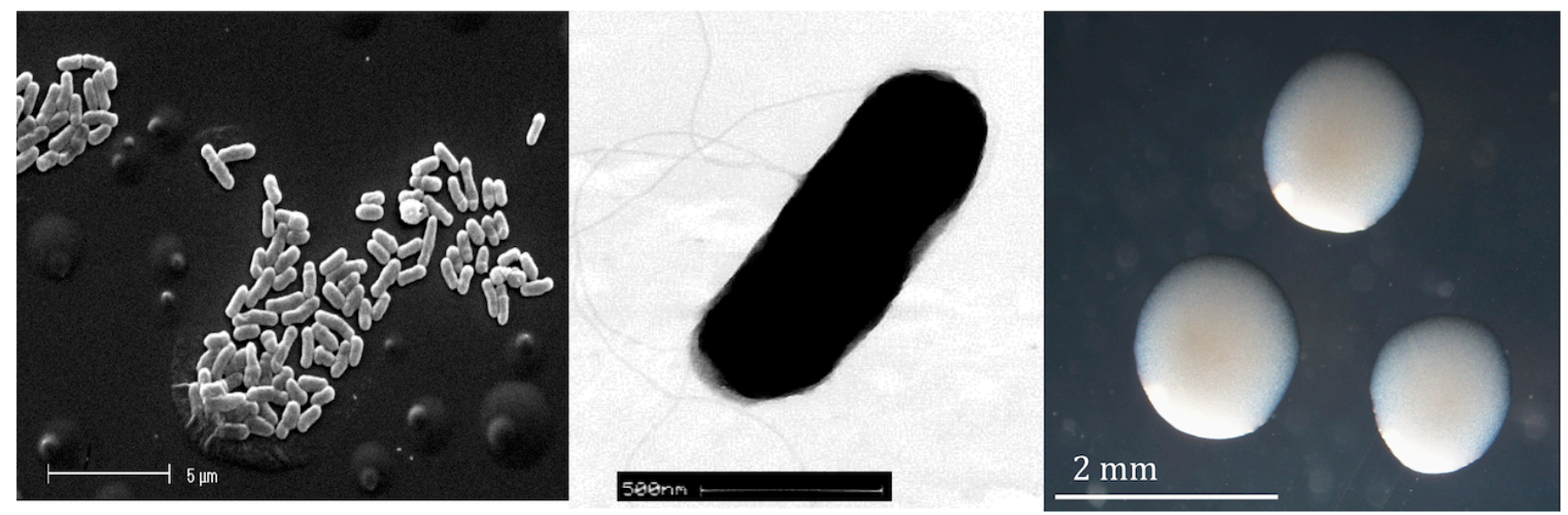

Figure 1. Images of Rhizobium leguminosarum bv. trifolii strain WSM1689 using scanning (Left) and transmission (Center) electron microscopy and the appearance of colony morphology on $1 / 2 \mathrm{LA}$ (Right). 
Table 1. Classification and general features of Rhizobium legum inosarum bv. trifolii strain WSM1689 according to the MIGS recommendations $[10,11]$.

\begin{tabular}{|c|c|c|c|}
\hline MIGS ID & Property & Term & Evidence code \\
\hline & \multirow{8}{*}{ Current classification } & Domain Bacteria & TAS [11] \\
\hline & & Phylum Proteobacteria & TAS [12] \\
\hline & & Class Alphaproteobacteria & TAS $[13,14]$ \\
\hline & & Order Rhizobiales & TAS $[14,15]$ \\
\hline & & Family Rhizobiaceae & TAS $[16,17]$ \\
\hline & & Genus Rhizobium & TAS $[16,18-21]$ \\
\hline & & Species Rhizobium leguminosarum bv. trifolii & TAS $[16,18,21,22]$ \\
\hline & & Strain WSM1689 & TAS [4] \\
\hline & Gram stain & Negative & IDA \\
\hline & Cell shape & Rod & IDA \\
\hline & Motility & Motile & IDA \\
\hline & Sporulation & Non-sporulating & NAS \\
\hline & Temperature range & Mesophile & NAS \\
\hline & Optimum temperature & $28^{\circ} \mathrm{C}$ & NAS \\
\hline & Salinity & Not reported & NAS \\
\hline \multirow[t]{3}{*}{ MIGS-22 } & Oxygen requirement & Aerobic & TAS [4] \\
\hline & Carbon source & Varied & NAS \\
\hline & Energy source & Chemoorg anotroph & NAS \\
\hline MIGS-6 & Habitat & Soil, root nodule, host & TAS [4] \\
\hline MIGS-15 & Biotic relationship & Free living, symbiotic & TAS [4] \\
\hline \multirow[t]{3}{*}{ MIGS-14 } & Pathogenicity & Non-pathogenic & NAS \\
\hline & Biosafety level & 1 & NAS [23] \\
\hline & Isolation & Root nodule & TAS [4] \\
\hline MIGS-4 & Geographic location & Naxos, Greece & IDA \\
\hline MIGS-5 & Nodule collection date & 1995 & IDA \\
\hline MIGS-4. 1 & Latitude & 37.128333 & IDA \\
\hline MIGS-4.2 & Long itude & 25.443333 & IDA \\
\hline MIGS-4. 3 & Depth & Not reported & \\
\hline MIGS-4.4 & Altitude & Not reported & \\
\hline
\end{tabular}

Evidence codes - IDA: Inferred from Direct Assay; TAS: Traceable Author Statement (i.e., a direct report exists in the literature); NAS: Non-traceable Author Statement (i.e., not directly observed for the living, isolated sample, but based on a generally accepted property for the species, or anecdotal evidence). These evidence codes are from the Gene Ontology project [24]. 


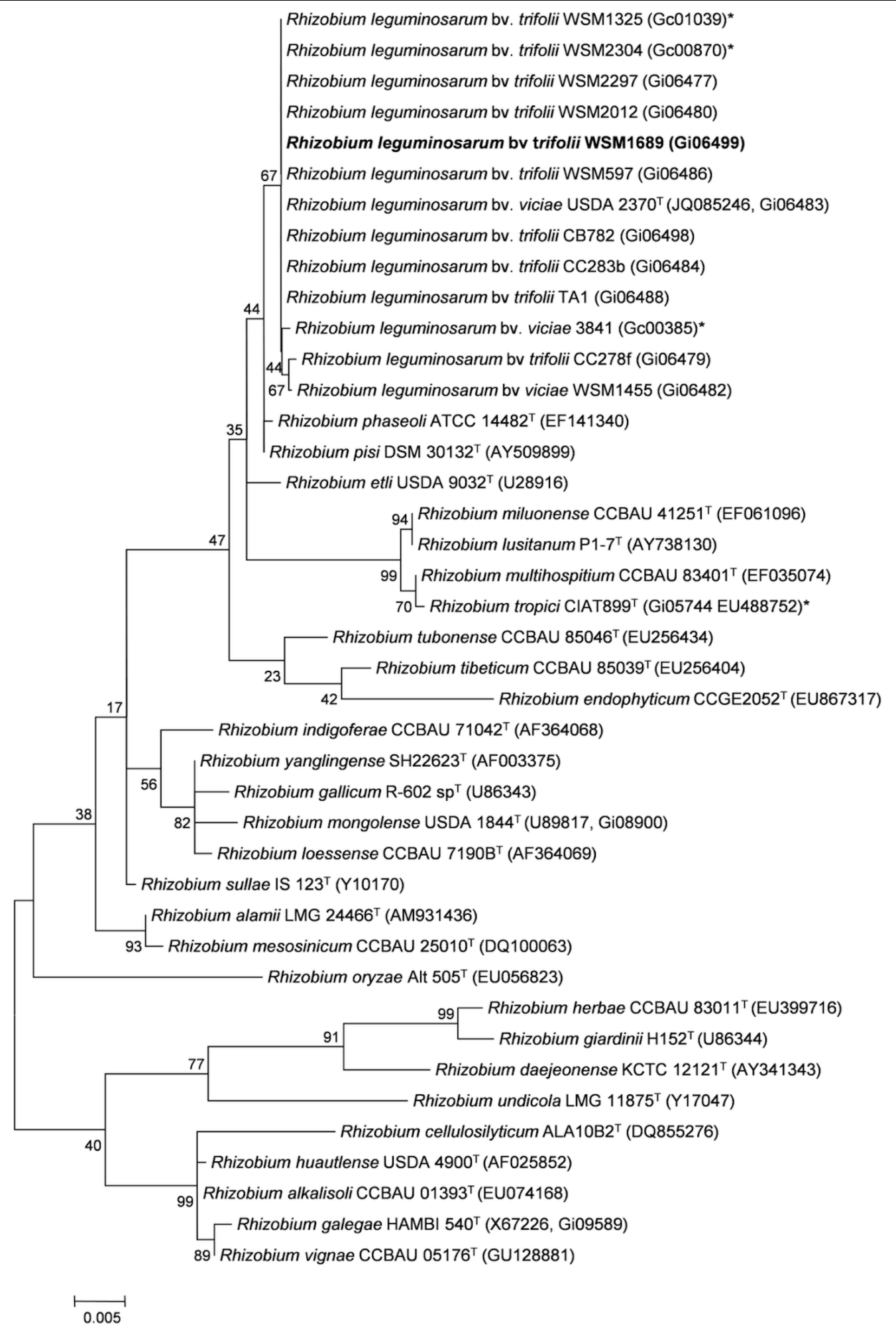

Figure 2. Phylogenetic tree showing the relationship of Rhizobium leguminosarum bv trifolii WSM1689 (shown in bold print) to other root nodulating Rhizobium spp. in the order Rhizobiales based on aligned sequences of the $16 \mathrm{~S}$ rRNA gene (1,180 bp internal region). All positions containing gaps and missing data were eliminated. All sites were informative and there were no gapcontaining sites. Phylogenetic analyses were performed using MEGA, version 5 [25]. The tree was built using the Maximum-Likelihood method with the General Time Reversible model [26]. Bootstrap analysis [27] with 500 replicates was performed to assess the support of the clusters. Type strains are indicated with a superscript T. Brackets after the strain name contain a DNA database accession number and/or a GOLD ID (beginning with the prefix $\mathrm{G}$ ) for a sequencing project reg istered in GOLD [28]. Published genomes are indicated with an asterisk. 
Table 2. Compatibility of WSM1689 with both perennial and annual Trifolium genotypes for nodulation (Nod) and $\mathrm{N}_{2}$-Fixation (Fix). Data compiled from [4].

\begin{tabular}{|c|c|c|c|c|c|c|}
\hline Species Name & Cultivar & Origin & Growth habit & Nod & Fix & Comment \\
\hline T. uniflorum & $\mathrm{Nil}$ & Europe & Perennial & Nod & Fix & Hig hly effective \\
\hline T. tumens & 1986267 & Europe & Perennial & Nod & Fix & Ineffective \\
\hline T. tumens & 16758246 & Europe & Perennial & Nod & Fix & Ineffective \\
\hline T. medium & 21881154 & Europe & Perennial & Nod & Fix & Ineffective \\
\hline T. repens & 037701 & Europe & Perennial & Nod & Fix & Ineffective \\
\hline T. repens & 036120 & Europe & Perennial & Nod & Fix & Ineffective \\
\hline T. pratense & Russian no 9 & Europe & Perennial & Nod & Fix & Ineffective \\
\hline T. pratense & Redquin & Europe & Perennial & Nod & Fix & Ineffective \\
\hline T. ambiguum & Endura & Europe & Perennial & Nod & Fix & Ineffective \\
\hline T. canescens & PL4188661999 & Europe & Perennial & Nod & Fix & Ineffective \\
\hline T. fragiferum & C1212 & Europe & Perennial & Nod & & No nodulation \\
\hline T. polymorphum & 87102 & South America & Perennial & Nod & Fix & Ineffective \\
\hline T. longipes & A2 436817 & North America & Perennial & Nod & & No nodulation \\
\hline T. subterraneum & York & Europe & Annual & Nod & Fix & Ineffective \\
\hline T. glanduliferum & CP187182 & Europe & Annual & Nod & Fix & Ineffective \\
\hline T. mulinerve & 87259 & Africa & Annual & Nod & & No nodulation \\
\hline T. tridentatum & CQ1263 & North America & Annual & Nod & Fix & Ineffective \\
\hline
\end{tabular}

\section{Symbiotaxonomy}

R. leguminosarum bv. trifolii WSM1689 is a highly effective microsymbiont of the perennial Eurasian clover Trifolium uniflorum (Table 2). In contrast, WSM1689 does not nodulate the perennial $T$. fragiferum and forms white ineffective (Fix-) nodules with other perennial and annual clovers of Eurasian origin. Moreover, WSM1689 is either Nod- or Fix- on clovers of North American or African origin. Therefore, WSM1689 is unusual in having an extremely narrow clover host range for the establishment of effective $\mathrm{N}_{2}$-fixing symbiosis.

\section{Genome sequencing and annotation Genome project history}

This organism was selected for sequencing on the basis of its environmental and agricultural relevance to issues in global carbon cycling, alternative energy production, and biogeochemical importance, and is part of the Community Sequencing Program at the U.S. Department of Energy, Joint Genome Institute (JGI) for projects of relevance to agency missions. The genome project is deposited in the Genomes OnLine Database [28] and a finished genome sequence in IMG/GEBA. Sequencing, finishing and annotation were performed by the JGI. A summary of the project information is shown in Table 3.

\section{Growth conditions and DNA isolation}

Rhizobium leguminosarum bv. trifolii strain WSM1689 was grown to mid logarithmic phase in TY rich medium on a gyratory shaker at $28^{\circ} \mathrm{C}$ [29]. DNA was isolated from $60 \mathrm{~mL}$ of cells using a CTAB (Cetyl trimethyl ammonium bromide) bacterial genomic DNA isolation method [30]. 
Table 3. Genome sequencing project information for Rhizobium leguminosarum bv. trifolii strain WSM1689.

\begin{tabular}{lll}
\hline MIGS ID & Property & Term \\
\hline MIGS-31 & Finishing quality & Finished \\
MIGS-2 8 & Libraries used & Illumina GAii shotgun and paired end 454 libraries \\
MIGS-29 & Sequencing platforms & Illumina GAii and 454 GS FLX Titanium technologies \\
MIGS-31.2 & Sequencing coverage & $8.3 x$ 454, 774.6x Illumina \\
MIGS-30 & Assemblers & VELVET, version 1.1.05; Newbler, version 2.6; phrap, version SPS - 4.24 \\
MIGS-32 & Gene calling methods & Prodigal 1.4, GenePRIMP \\
& Genbank ID & Not yet available \\
& Genbank Date of Release & Not yet released \\
& GOLD ID & Gi06499 \\
& NCBI project ID & 62289 \\
& Database: IMG-GEBA & 2510065019 \\
& Project relevance & Symbiotic nitrogen fixation, agriculture \\
\hline
\end{tabular}

\section{Genome sequencing and assembly}

The genome of Rhizobium leguminosarum bv. trifolii strain WSM1689 was sequenced at the Joint Genome Institute (JGI) using a combination of Illumina [31] and 454 technologies [32]. An Illumina GAii shotgun library which generated 73,565,648 reads totaling 5,591 $\mathrm{Mbp}$, and a paired end 454 library with an average insert size of 12 Kbp which generated 376,185 reads totaling 93.4 Mbp of 454 data were generated for this genome. All general aspects of library construction and sequencing performed at the JGI can be found at [30]. The initial draft assembly contained 100 contigs in 4 scaffolds. The 454 paired end data was assembled with Newbler, version 2.6. The Newbler consensus sequences were computationally shredded into 2 $\mathrm{Kbp}$ overlapping fake reads (shreds). Illumina sequencing data was assembled with VELVET, version 1.1.05 [33], and the consensus sequence computationally shredded into $1.5 \mathrm{Kbp}$ overlapping fake reads (shreds). We integrated the 454 Newbler consensus shreds, the Illumina VELVET consensus shreds and the read pairs in the 454 paired end library using parallel phrap, version SPS - 4.24 (High Performance Software, LLC). The software Consed [34-36] was used in the following finishing process. Illumina data was used to correct potential base errors and increase consensus quality using the software Polisher developed at JGI (Alla Lapidus, unpublished). Possible misassemblies were corrected using gapResolution (Cliff Han, unpublished), Dupfinisher [37], or sequencing cloned bridging PCR fragments with subcloning. Gaps between contigs were closed by editing in Consed, by PCR and by Bubble PCR (J-F Cheng, unpublished) primer walks. A total of 93 additional reactions were necessary to close gaps and to raise the quality of the finished sequence.
The total genome size is $6.9 \mathrm{Mbp}$ and the final assembly is based on $57.3 \mathrm{Mbp}$ of 454 draft data which provides an average $8.3 \times$ coverage of the genome and 5,345 Mbp of Illumina draft data which provides an average $774.6 \times$ coverage of the genome.

\section{Genome annotation}

Genes were identified using Prodigal [38] as part of the DOE-JGI genome annotation pipeline, followed by a round of manual curation using the JGI GenePRIMP pipeline [39]. The predicted CDSs were translated and used to search the National Center for Biotechnology Information (NCBI) nonredundant database, UniProt, TIGRFam, Pfam, PRIAM, KEGG, COG, and InterPro databases. These data sources were combined to assert a product description for each predicted protein. Non-coding genes and miscellaneous features were predicted using tRNAscan-SE [40], RNAMMer [41], Rfam [42], TMHMM [43], and SignalP [44]. Additional gene prediction analyses and functional annotation were performed within the Integrated Microbial Genomes (IMG-ER) platform $[45,46]$.

\section{Genome properties}

The genome is 6,903,379 nucleotides with $60.94 \%$ GC content (Table 4 and Figures 3a,3b,3c,3d,3e and Figure 3f), and comprised of 6 replicons. From a total of 6,798 genes, 6,709 were protein encoding and 89 RNA only encoding genes. Within the genome, 206 pseudogenes were also identified. The majority of genes (79.52\%) were assigned a putative function whilst the remaining genes were annotated as hypothetical. The distribution of genes into COGs functional categories is presented in Table 5 . 
Table 4. Genome Statistics for Rhizobium leguminosarum bv. trifolii strain WSM1689.

\begin{tabular}{lrr}
\hline Attribute & Value & \% of Total \\
\hline Genome size (bp) & $6,903,379$ & 100.00 \\
DNA coding region (bp) & $6,004,795$ & 86.98 \\
DNA G+C content (bp) & $4,206,909$ & 60.94 \\
Number of replicons & 6 & \\
Total genes & 6,798 & 100.00 \\
RNA genes & 89 & 1.31 \\
Protein-coding genes & 6,709 & 98.69 \\
Genes with function prediction & 5,406 & 79.52 \\
Genes assigned to COGs & 5,400 & 79.44 \\
Genes assigned Pfam domains & 5,618 & 82.64 \\
Genes with signal peptides & 591 & 8.69 \\
Genes coding transmembrane proteins & 1,524 & 22.42 \\
CRISPR repeats & 0 & \\
\hline
\end{tabular}

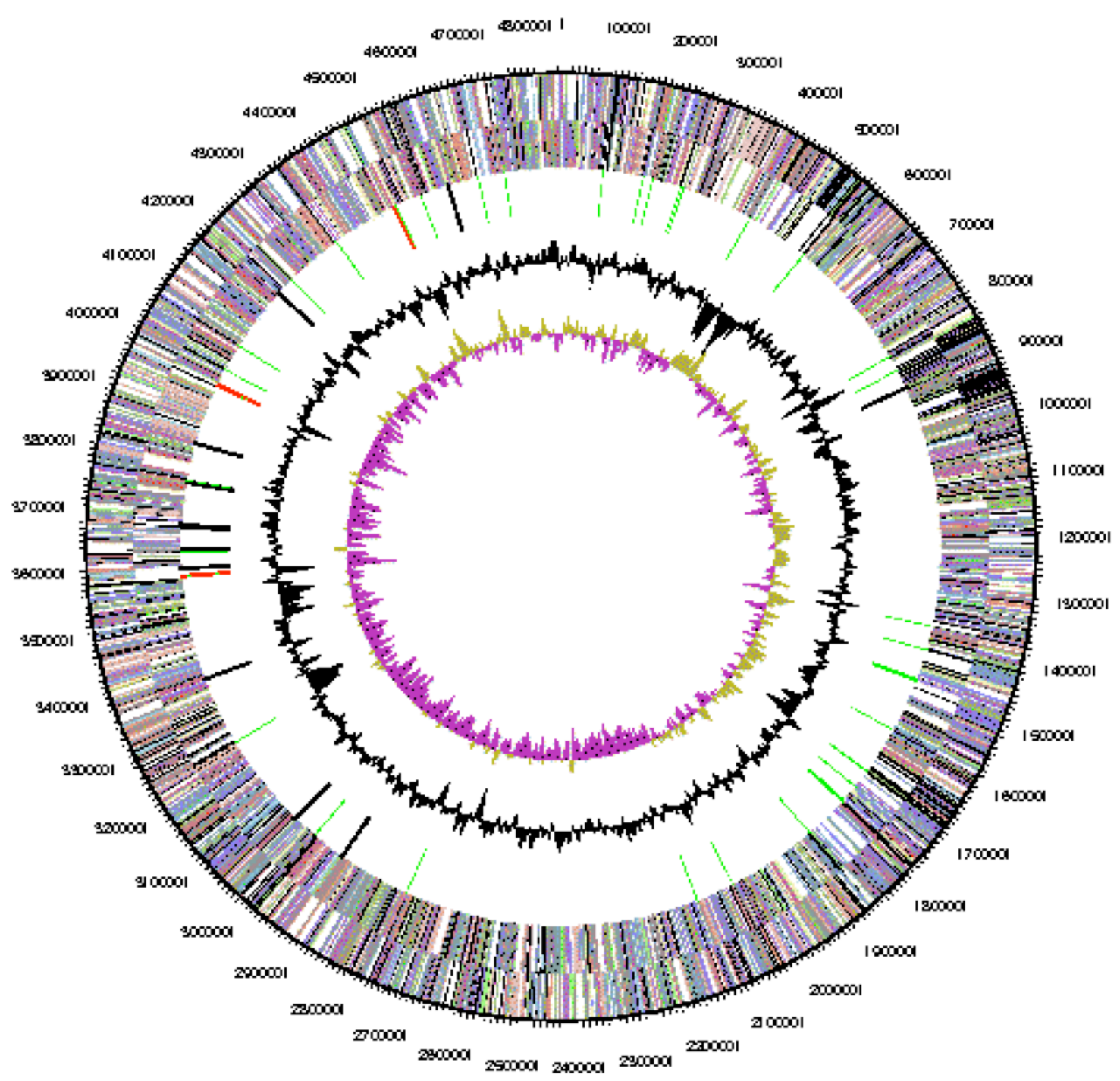

Figure 3a. Graphical circular map of Replicon WSM1689_Rleg3_Contig1814.1 of the Rhizobium leguminosarum bv. trifolii strain WSM1689 genome. From outside to the center: Genes on forward strand (color by COG categ ories as denoted by the IMG platform), Genes on reverse strand (color by COG categories), RNA genes (tRNAs g reen, sRNAs red, other RNAs black), GC content, GC skew. 


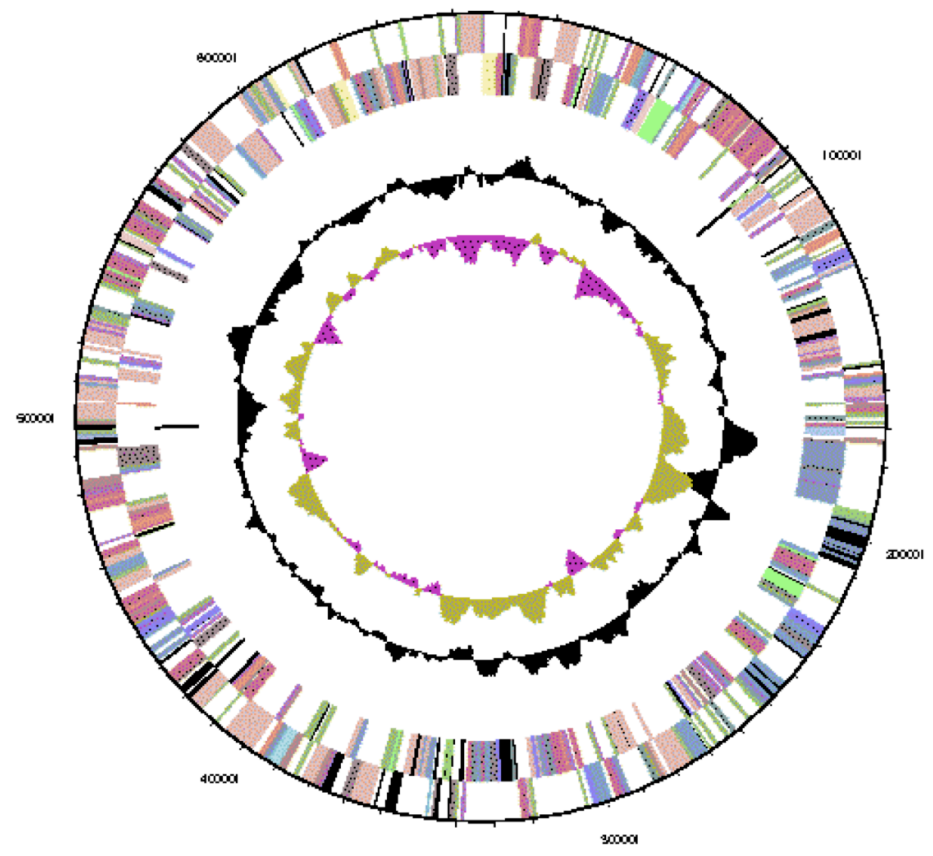

Figure 3b. Graphical circular map of replicon WSM1689_Rleg 3_Contig1813.2 of the Rhizobium leguminosarum bv. trifolii strain WSM1689 genome. From outside to the center: Genes on forward strand (color by COG categ ories as denoted by the IMG platform), Genes on reverse strand (color by COG categories), RNA genes (tRNAs green, sRNAs red, other RNAs black), GC content, GC skew.

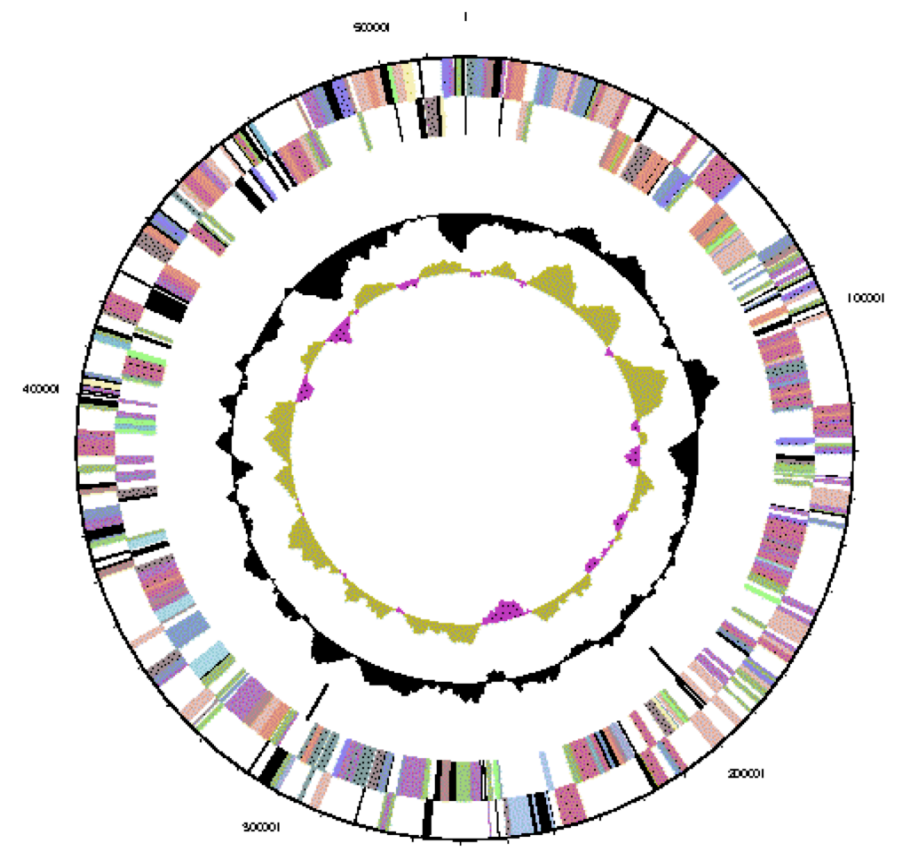

Figure 3c. Graphical circular map of replicon WSM1689_Rleg3_Contig1812.3 of the Rhizobium leguminosarum bv. trifolii strain WSM1689 genome. From outside to the center. Genes on forward strand (color by COG categories as denoted by the IMG platform), Genes on reverse strand (color by COG categories), RNA genes (tRNAs green, sRNAs red, other RNAs black), GC content, GC skew. 


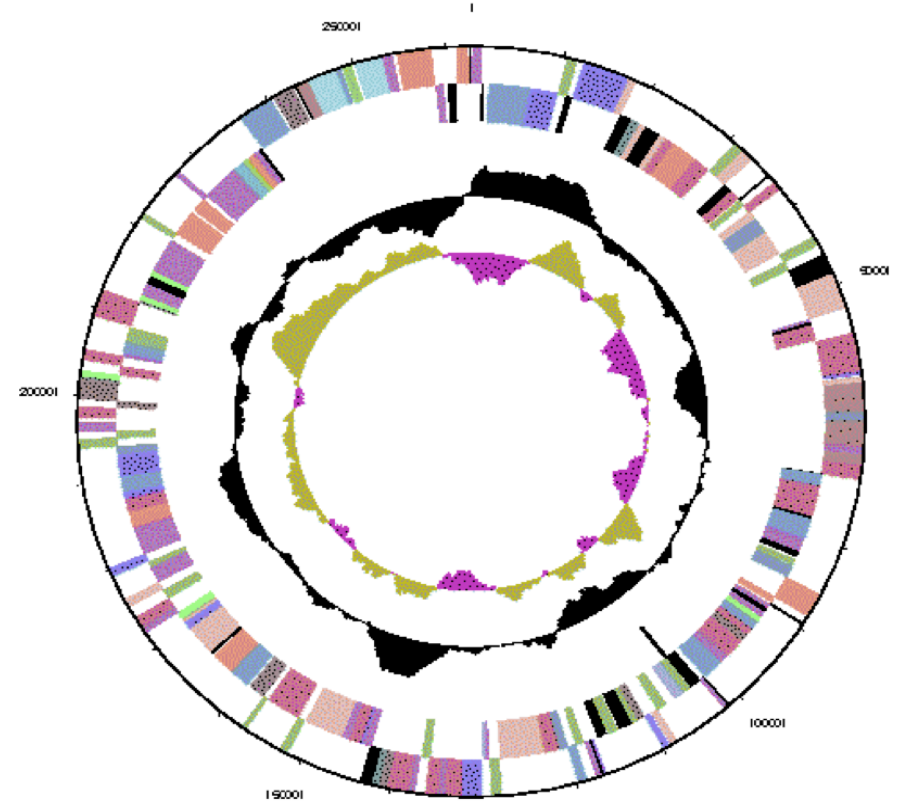

Figure 3d. Graphical circular map of replicon WSM1689_Rleg3_Contig1810.5 of the Rhizobium leguminosarum bv. trifolii strain WSM1689 genome. From outside to the center. Genes on forward strand (color by COG categories as denoted by the IMG platform), Genes on reverse strand (color by COG categories), RNA genes (tRNAs green, sRNAs red, other RNAs black), GC content, GC skew.

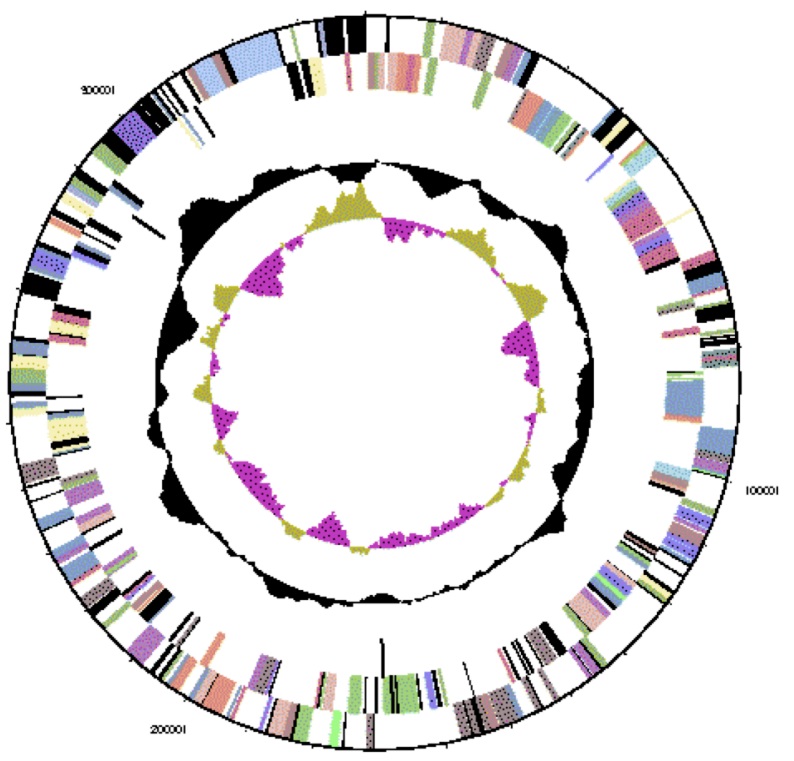

Figure 3e. Graphical circular map of replicon WSM1689_Rleg3_Contig1811.4 of the Rhizobium leguminosarum bv. trifolii strain WSM1689 genome. From outside to the center: Genes on forward strand (color by COG categories as denoted by the IMG platform), Genes on reverse strand (color by COG categories), RNA genes (tRNAs green, sRNAs red, other RNAs black), GC content, GC skew. 


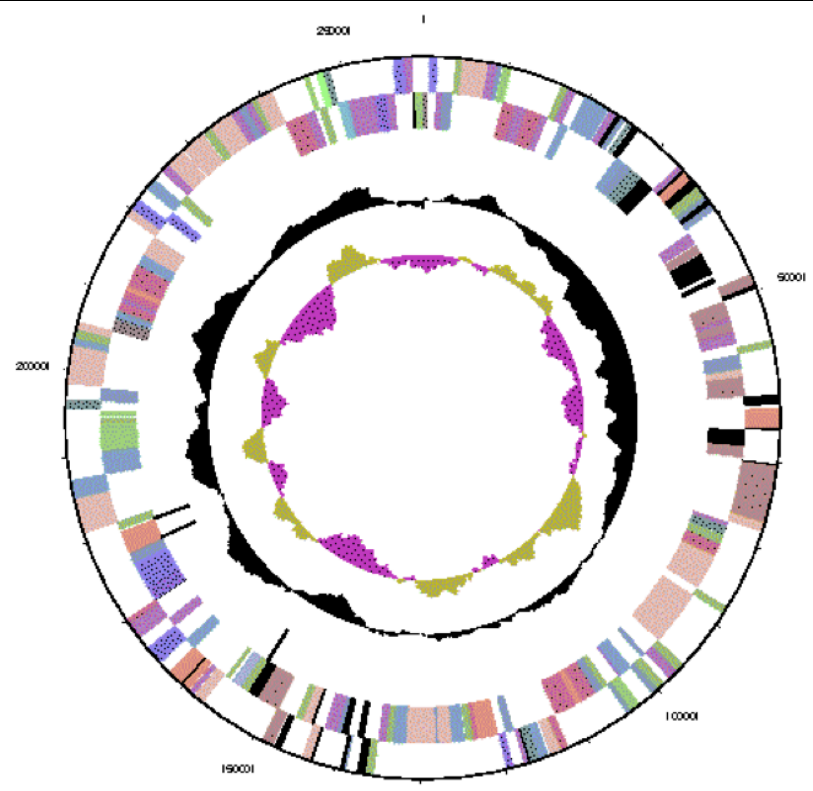

Figure 3f. Graphical circular map of replicon WSM1689_Rleg3_Contig1809.6 of the Rhizobium leguminosarum bv. trifolii strain WSM1689 genome. From outside to the center: Genes on forward strand (color by COG categories as denoted by the IMG platform), Genes on reverse strand (color by COG categories), RNA genes (tRNAs green, sRNAs red, other RNAs black), GC content, GC skew.

Table 5. Number of protein coding genes of Rhizob ium le gum inosarum bv. trifolii strain WSM1689 associated with the general COG functional categ ories.

\begin{tabular}{crrl}
\hline Code & Value & \%age & COG Category \\
\hline J & 205 & 3.40 & Translation, ribosomal structure and biogenesis \\
A & 0 & 0.00 & RNA processing and modification \\
K & 581 & 9.62 & Transcription \\
L & 153 & 2.53 & Replication, recombination and repair \\
B & 2 & 0.03 & Chromatin structure and dynamics \\
D & 39 & 0.65 & Cell cycle control, mitosis and meiosis \\
Y & 0 & 0.00 & Nuclear structure \\
V & 66 & 1.09 & Defense mechanisms \\
T & 311 & 5.15 & Signal transduction mechanisms \\
M & 329 & 5.45 & Cell wall/membrane biog enesis \\
N & 81 & 1.34 & Cell motility \\
Z & 0 & 0.00 & Cytoskeleton \\
W & 0 & 0.00 & Extracellular structures \\
U & 82 & 1.36 & Intracellular trafficking and secretion \\
O & 187 & 3.10 & Posttranslational modification, protein turnover, chaperones \\
C & 311 & 5.15 & Energy production conversion \\
\hline
\end{tabular}


Table 5 (cont.). Number of protein coding genes of Rhizobium legum inosarum bv. trifolii strain WSM1689 associated with the general COG functional categ ories.

\begin{tabular}{crrl}
\hline Code & \multicolumn{1}{c}{ Value } & \%age & COG Category \\
\hline G & 683 & 11.31 & Carbohydrate transport and metabolism \\
E & 629 & 10.42 & Amino acid transport metabolism \\
F & 105 & 1.74 & Nucleotide transport and metabolism \\
H & 192 & 3.18 & Coenzyme transport and metabolism \\
I & 222 & 3.68 & Lipid transport and metabolism \\
P & 297 & 4.92 & Inorganic ion transport and metabolism \\
Q & 147 & 2.43 & Secondary metabolite biosynthesis, transport and catabolism \\
R & 795 & 13.17 & General function prediction only \\
S & 620 & 10.27 & Function unknown \\
- & 1,398 & 20.56 & Not in COGS \\
- & 6,037 & - & Total \\
\hline
\end{tabular}

\section{Acknowledgements}

This work was performed under the aus pices of the US Department of Energy's Office of Science, Biological and Environmental Research Program, and by the University of California, Lawrence Berkeley National Laboratory under contract No. DE-AC02-05CH11231, Lawrence Livermore National Laboratory under Contract No. DE-

\section{References}

1. Terpolilli JJ, Hood GA, Poole PS. What determines the efficiency of $\mathrm{N}_{2}$-fixing RhizobiumLeg ume symbioses? Adv Microb Physiol 2012; 60:325-389. PubMed

http://dx.doi.org/10.1016/B978-0-12-3982643.00005-X

2. Howieson JG, O'Hara GW, Carr SJ. Changing roles for leg umes in Mediterranean ag riculture: developments from an Australian perspective. Field Crops Res 2000; 65:107-122. http://dx.doi.org/10.1016/S0378-4290(99)00081$\underline{7}$

3. Lamont EJ, Zoghlami A, Hamilton RS, Bennett SJ. Clovers (Trifolium L.). In: Maxted N, Bennett SJ, editors. Plant Genetic Resources of Leg umes in the Mediterranean. Dordrecht: Kluwer Academic Publishers; 2001. p 79-98.

4. Howieson J, Yates R, O'Hara G, Ryder M, Real D. The interactions of Rhizobium leguminosarum biovar trifolii in nodulation of annual and perennial Trifolium spp from diverse centres of orig in. Aust J Exp Agric 2005; 45:199-207. http://dx.doi.org/10.1071/EA03167
AC52-07NA27344, and Los Alamos National Laboratory under contract No. DE-AC02-06NA25396. We gratefully acknowledge the funding received from the Murdoch University Sir Walter Murdoch Adjunct Professor Scheme for Professor Philip Poole.

5. Reeve W, O'Hara G, Chain P, Ardley J, Brau L, Nandesena K, Tiwari R, Copeland A, Nolan M, Han C, et al. Complete genome sequence of Rhizobium leguminosarum bv. trifolii strain WSM1325, an effective microsymbiont of annual Mediterranean clovers. Stand Genomic Sci 2010; 2:347-356. PubMed http://dx.doi.org/10.4056/sigs.852027

6. Reeve W, O'Hara G, Chain P, Ardley J, Brau L, Nandesena K, Tiwari R, Malfatti S, Kiss H, Lapidus A, et al. Complete genome sequence of Rhizobium le guminosarum bv trifolii strain WSM2304, an effective microsymbiont of the South American clover Trifolium polymorphum. Stand Genomic Sci 201 0; 2:66-76. PubMed http://dx.doi.org/10.4056/sigs.44642

7. Howieson JG, Ewing MA, D'antuono MF. Selection for acid tolerance in Rhizobium meliloti. Plant Soil 1988; 105:179-188. http://dx.doi.org/10.1007/BF02 376781

8. Bering er JE. R factor transfer in Rhizobium leguminosarum. J Gen Microbiol 1974; 84:188198. PubMed http://dx.doi.org/10.1099/00221287-84-1-188 
9. Terpolilli JJ. Why are the symbioses between some genotypes of Sinorhizobium and Medicago suboptimal for $\mathrm{N}_{2}$ fixation? Perth: Murdoch University; 2009. 223 p.

10. Field D, Garrity G, Gray T, Morrison N, Seleng ut J, Sterk P, Tatusova T, Thomson N, Allen M, Angiuoli SV, et al. Towards a richer description of our complete collection of genomes and metagenomes "Minimum Information about a Genome Sequence " (MIGS) specification. Nat Biotechno/ 2008; 26:541-547. PubMed http://dx.doi.org/10.1038/nbt1360

11. Woese CR, Kandler O, Wheelis ML. Towards a natural system of organisms: proposal for the domains Archaea, Bacteria, and Eucarya. Proc Natl Acad Sci USA 1990; 87:4576-4579. PubMed http://dx.doi.org/10.1073/pnas.87.12.4576

12. Garrity GM, Bell JA, Lilburn T. Phylum XIV. Proteobacteria phyl. nov. In: Garrity GM, Brenner DJ, Krieg NR, Staley JT (eds), Bergey's Manual of Systematic Bacteriology, Second Edition, Volume 2, Part B, Springer, New York, 2005, p. 1.

13. Garrity GM, Bell JA, Lilburn T. Class I. Alphaproteobacteria class. nov. In: Garrity GM, Brenner DJ, Krieg NR, Staley JT (eds), Bergey's Manual of Systematic Bacteriology, Second Edition, Volume 2, Part C, Springer, New York, 2005, p. 1.

14. Validation List No. 107. List of new names and new combinations previously effectively, but not validly, published. Int I Syst Evol Microbiol 2006; 56:1-6. PubMed http://dx.doi.org/10.1099/ijs.0.64188-0

15. Kuykendall LD. Order VI. Rhizobiales ord. nov. In: Garrity GM, Brenner DJ, Kreig NR, Staley JT, editors. Bergey's Manual of Systematic Bacteriology. Second ed: New York: Spring er - Verlag; 2005. p 324.

16. Skerman VBD, McGowan V, Sneath PHA. Approved Lists of Bacterial Names. Int J Syst Bacteriol 1980; 30:225-420. http://dx.doi.org/10.1099/00207713-30-1-225

17. Conn HJ. Taxonomic relationships of certain nonsporeforming rods in soil. / Bacterio/ 1938; 36:320-321.

18. Frank B. Über die Pilzsymbiose der Leguminosen. Ber Dtsch Bot Ges 1889; 7:332-346.

19. Jordan DC, Allen ON. Genus I. Rhizobium Frank 1889, 338; Nom. gen. cons. Opin. 34, Jud. Comm. 1970, 11. In: Buchanan RE, Gibbons NE (eds), Bergey's Manual of Determinative Bacteri- ology, Eighth Edition, The Williams and Wilkins Co., Baltimore, 1974, p. 262-264.

20. Young JM, Kuykendall LD, Martínez-Romero E, Kerr A, Sawada H. A revision of Rhizobium Frank 1889 , with an emended description of the genus, and the inclusion of all species of Agrobacterium Conn 1942 and Allorhizobium undicola de Lajudie et al. 1998 as new combinations: Rhizobium radiobacter, $R$. rhizogenes, $R$. rubi, $R$. undic ola and R. vitis. Int I Syst Evol Microbiol 2001; 51:89-103. PubMed

21. Editorial Secretary (for the Judicial Commission of the International Committee on Nomenclature of Bacteria). OPINION 34: Conservation of the Generic Name Rhizobium Frank 1889. Int J Syst Bacteriol 1970; 20:11-12. http://dx.doi.org/10.1099/00207713-20-1-11

22. Ramírez-Bahena $M H$, García-Fraile $P$, Peix $A$, Valverde A, Rivas R, Igual JM, Mateos PF, Martínez-Molina E, Velázquez E. Revision of the taxonomic status of the species Rhizobium legum inosarum (Frank 1879) Frank 1889AL, Rhizobium phaseoli Dangeard 1926AL and Rhizobium trifolii Dangeard 1926AL. R. trifolii is a later synonym of $R$. leguminosarum. Reclassification of the strain $R$. leguminosarum DSM 30132 (=NCIMB 11478) as Rhizobium pisisp. nov. Int J Syst Evol Microbiol 2008; 58:2484-2 490. PubMed http://dx.doi.org/10.1099/ijs.0.65621-0

23. Agents B. Technical rules for biological agents. TRBA (http://www.baua.de):466.

24. Ashburner M, Ball CA, Blake JA, Botstein D, Butler H, Cherry JM, Davis AP, Dolinski K, Dwight SS, Eppig JT, et al. Gene ontology: tool for the unification of biology. The Gene Ontology Consortium. Nat Genet 2000; 25:25-29. PubMed http://dx.doi.org/10.1038/75556

25. Tamura K, Peterson D, Peterson N, Stecher G, Nei M, Kumar S. MEGA5: Molecular Evolutionary Genetics Analysis using Maximum Likelihood, Evolutionary Distance, and Maximum Parsimony Methods. Mol Biol Evol 2011; 28:2731-2739. PubMed http://dx.doi.org/10.1093/molbev/msr121

26. Nei M, Kumar S. Molecular Evolution and Phylog enetics. New York: Oxford University Press; 2000.

27. Felsenstein J. Confidence limits on phylog enies: an approach using the bootstrap. Evolution 1985; 39:783-791. http://dx.doi.org/10.2307/2408678

28. Liolios K, Mavromatis K, Tavernarakis N, Kyrpides NC. The Genomes On Line Database (GOLD) in 
2007: status of genomic and metag enomic projects and their associated metadata. Nucleic Acids Res 2008; 36:D475-D479. PubMed http://dx.doi.org/10.1093/nar/gkm884

29. Reeve WG, Tiwari RP, Worsley PS, Dilworth MJ, Glenn AR, Howieson JG. Constructs for insertional mutagenesis, transcriptional signal localization and gene regulation studies in root nodule and other bacteria. Microbiology 1999; 145:1307-1316. PubMed http://dx.doi.org/10.1099/13500872-145-6-1307

30. DOE Joint Genome Institute. http://my.jgi.doe.gov/g eneral/index.html

31. Bennett S. Solexa Ltd. Pharmacogenomics 2004; 5:433-438. PubMed http://dx.doi.org/10.1517/14622416.5.4.433

32. Margulies $M$, Eg holm M, Altman WE, Attiya $S$, Bader JS, Bemben LA, Berka J, Braverman MS, Chen YJ, Chen Z, et al. Genome sequencing in microfabricated hig h-density picolitre reactors. Nature 2005; 437:376-380. PubMed

33. Zerbino DR. Using the Velvet de novo assembler for short-read sequencing technolog ies. Current Protocols in Bioinformatics 2010; Chapter 11:Unit 115.

34. Ewing B, Green P. Base-calling of automated sequencer traces using phred. II. Error probabilities. Genome Res 1998; 8:186-194. PubMed http://dx.doi.org/10.1101/gr.8.3.175

35. Ewing B, Hillier L, Wendl MC, Green P. Basecalling of automated sequencer traces using phred. I. Accuracy assessment. Genome Res 1998; 8:175-1 85. PubMed http://dx.doi.org/10.1101/gr.8.3.175

36. Gordon D, Abajian C, Green P. Consed: a graphical tool for sequence finishing. Genome Res

1998; 8:195-202. PubMed http://dx.doi.org/10.1101/gr.8.3.195

37. Han C, Chain P. Finishing repeat reg ions automatically with Dupfinisher. In: Valafar HRAH, editor. Proceeding of the 2006 international conference on bioinformatics \& computational biology: CSREA Press; 2006. p 141-146.
38. Hyatt D, Chen GL, Locascio PF, Land ML, Larimer FW, Hauser LJ. Prodig al: prokaryotic gene recog nition and translation initiation site identification. BMC Bioinformatics 2010; 11:119. PubMed http://dx.doi.org/10.1186/1471-2105-11-119

39. Pati A, Ivanova NN, Mikhailova N, Ovchinnikova G, Hooper SD, Lykidis A, Kypides NC.

GenePRIMP: a gene prediction improvement pipeline for prokaryotic genomes. Nat Methods 2010; 7:455-457. PubMed http://dx.doi.org/10.1038/nmeth.1457

40. Lowe TM, Eddy SR. tRNAscan-SE: a prog ram for improved detection of transfer RNA genes in genomic sequence. Nucleic Acids Res 1997; 25:955-964. PubMed

41. Lagesen K, Hallin P, Rodland EA, Staerfeldt HH, Rognes T, Ussery DW. RNAmmer: consistent and rapid annotation of ribosomal RNA genes. Nucleic Acids Res 2007; 35:3100-3108. PubMed http://dx.doi.org/10.1093/nar/gkm160

42. Griffiths-Jones S, Bateman A, Marshall M, Khanna A, Eddy SR. Rfam: an RNA family database. Nucleic Acids Res 2003; 31:439-441. PubMed http://dx.doi.org/10.1093/nar/g kg006

43. Krogh A, Larsson B, von Heijne G, Sonnhammer EL. Predicting transmembrane protein topology with a hidden Markov model: application to complete genomes. J Mol Biol 2001; 305:567580. PubMed http://dx.doi.org/10.1006/jmbi.2000.4315

44. Bendtsen JD, Nielsen H, von Heijne G, Brunak S. Improved prediction of sig nal peptides: Sig nalP 3.0. J Mol Biol 2004; 340:783-795. PubMed http://dx.doi.org/10.1016/j.jmb.2004.05.028

45. Markowitz VM, Mavromatis K, Ivanova NN, Chen IM, Chu K, Kyrpides NC. IMG ER: a system for microbial genome annotation expert review and curation. Bioinformatics 2009; 25:2271-2278. PubMed http://dx.doi.org/10.1093/bioinformatics/btp393

46. Integ rated Microbial Genomes (IMG-ER) platform. http://img.jgi.doe.gov/er 